\title{
Development of Physically Based Atomistic Microstructures: The Effect on the Mechanical Response of Polycrystals
}

\author{
Jacob Gruber ${ }^{\mathrm{a}, \mathrm{b}, *}$, Hojun Lim ${ }^{\mathrm{b}}$, Fadi Abdeljawad ${ }^{\mathrm{b}}$, Stephen Foiles ${ }^{\mathrm{b}}$, Garritt J. Tucker ${ }^{\mathrm{a}, * *}$ \\ ${ }^{a}$ Drexel University, 3141 Chestnut St, Philadelphia, PA 19104, USA \\ ${ }^{b}$ Sandia National Laboratories, PO Box 5800, MS 1411, Albuquerque, NM 87185, USA
}

\begin{abstract}
A method for the generation of atomistic realizations of polycrystalline aggregates from a phase field grain growth model is presented. Topologies of computational microstructures constructed from the proposed method as well as conventional Poisson Voronoi tessellation are quantitatively compared. While little difference is exhibited in the macroscale mechanical response, substantial differences in the resolved contribution to strain of deformation mechanisms of the structures under uniaxial tension are uncovered using post-processing kinematic metrics. These differences in the fundamental strain accommodation processes suggest that grain topology and grain boundary character significantly affect local responses of polycrystals in molecular dynamics simulations and that significant attention should be paid to the chosen starting microstructure.
\end{abstract}

Keywords: polycrystalline microstructure generation, molecular dynamics, mechanical properties

\section{Introduction}

Polycrystalline materials are agglomerations of many crystals, or grains, with mean grain size typically in the micron range for conventional metals and a few nanometers for nanocrystalline materials. The volume fraction of interfaces, i.e., grain boundaries (GBs), scales inversely with grain size [1] and therefore in nanocrystalline materials, a significant fraction of atoms reside in interfaces, driving a change in behavior and properties $[2,3,4,5]$. The onset of interfacemediated physics drives divergent behavior from coarse-grained systems, including enhanced strength and ductiliy $[6,7,8,9,10,11,12]$, improved fatigue resistance $[13,14,15,16]$, capacity for superplasticity $[17,18,19,20]$, and greater radiation tolerance [21, 22, 23], which has produced

\footnotetext{
* Corresponding author

** Principal corresponding author

Email addresses: jgruber@drexel.edu (Jacob Gruber), gtucker@coe.drexel.edu (Garritt J. Tucker)
} 
behavior of these systems is of particular interest; the conventional Hall-Petch model predicts increases in strength and hardness with decreasing grain size [27, 28], but recent findings suggest that nanocrystalline systems become softer $[29,30]$ due to a change in the dominant deformation mechanism from dislocation plasticity to grain boundary mediated deformation as grain size decreases below 10-20nm [31, 32].

In nano-grained materials, interfaces such as GBs begin to fundamentally govern material behavior. As such, significant effort has been made to better correlate structure to properties in nanoscale interfaces. However, the behavior of nanoscale interfaces is difficult to directly probe experimentally, but can be efficiently studied using atomistic simulations techniques such as molecular dynamics (MD). This computational method, in particular, has been used to investigate and provide pivotal insight into plasticity [33, 34, 35, 36, 37, 38], shock response [39, 40, 41], radiation tolerance $[42,43]$, solute segregation behavior [44, 45], and thermal conductivity [46, 47] both at isolated interfaces and in nanocrystalline systems.

Both atomistic and continuum scale simulation methods require the creation of computational microstructures. Polycrystals are commonly constructed using Voronoi tessellation methods $[48,49]$. The Voronoi diagram [50] is constructed for a distribution of grain centers, and the resulting polyhedra are taken as grains. The most common form of this method is Poisson Voronoi (PV) where grain centers are placed following a uniform distribution. Microstructures appear superficially similar to nanocrystalline structures produced in experiment (Fig 1), but differ in several critical regards. Voronoi constructions can produce grains with sharp points, wedge-shaped grains, or planar prismatic grains that are not observed experimentally. The grain topology of microstructures generated using PV has been shown to be significantly different than grain growth simulations and real systems [51]. Voronoi constructions also produce planar grain boundaries, while curved boundaries are common in experimentally observed microstructures. Triple junction morphology is also not accurately reproduced; thermodynamic theory predicts that triple junctions ought to have a dihedral angle of $2 \pi / 3[52,53]$ in isotropic media, but is not consistently reproduced by Voronoi constructions. Properties of nanocrystalline systems have been found to heavily depend on interface character $[54,55,56,57]$, therefore it is critical that realistic representations of grains and GBs are used as the initial conditions for simulations to more accurately predict the behavior of the microstructure under load.

Attempts have been made to improve Voronoi constructions to avoid some of these problems. So-called inverse Monte Carlo methods have been implemented to tune grain size distributions 
and misorientation angle distributions for use in molecular dynamics simulations [32, 58, 59].

Hardcore Voronoi tessellation, in which a minimum distance between grains is enforced, [60] ciated with the Voronoi construction. Idealized microstructures are often annealed to allow the relaxation of GBs, but this method is unreliable, computationally expensive and growth during annealing prevents direct control of grain size. Other methods of producing initial microstructures include the solidification of melts [61] and the sintering of finite sized particles [62]. The solidification method requires a very rapid quenching rate $\left(10^{12} \mathrm{~K} / \mathrm{s}\right)$, which produces an unrealistic concentration of twin boundaries; the sintering method often leaves a significant fraction of voids in the microstructure.

Phase field grain growth models, on the other hand, have been used to produce realistic microstructures, with curved boundaries and the correct triple junction dihedral angle [63]. In this framework, different phases of a material are designated by one or multiple 'order parameters' which evolve in time following thermodynamic and physical constraints. Phase field models have been shown to reproduce many aspects of real nanocrystalline microstructures, including grain boundary morphology and grain topology [63, 64, 58, 65].

In this paper, we present a method for discretizing microstructures from voxelated representations to atomistic resolution and quantitatively compare this structure to those produced by a PV method. While this work focuses on voxelated structures produced through phase field modeling, other methods such as Potts models or even experimental data from electron backscatter diffraction may be used. The mechanical properties, strain accommodation and deformation mechanisms are investigated with the aim of quantifying the differences produced by divergent GB morphology and grain topology.

\section{Methods}

\subsection{Phase Field Grain Growth Model}

A recently developed phase field model of grain growth is employed to generate polycrystalline aggregates $[66,65]$. Order parameters $(\mathrm{OPs}), \phi_{i}(\mathbf{r}, t), i=1 \cdots n$, define individual grain orientations, where $\mathbf{r}$ and $t$ are the positions in space and time and $n$ is then number of OPs. The free energy functional, $\mathcal{F}_{\text {tot }}$, is defined as

$$
\mathcal{F}_{\text {tot }}=\int d \mathbf{r}\left[W_{\phi} f_{\text {grain }}\left(\phi_{i}, \cdots, \phi_{n}\right)+\sum_{i}^{n_{\phi}} \frac{\epsilon^{2}}{2}\left|\nabla \phi_{i}\right|^{2}\right],
$$


a)

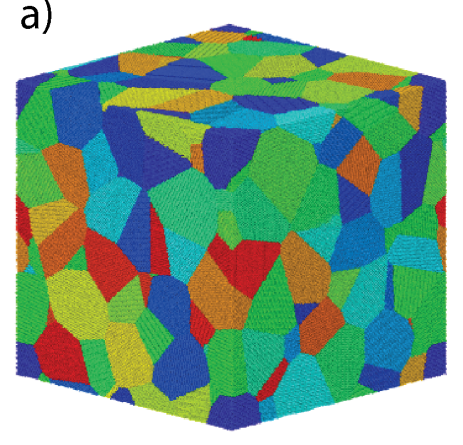

c)

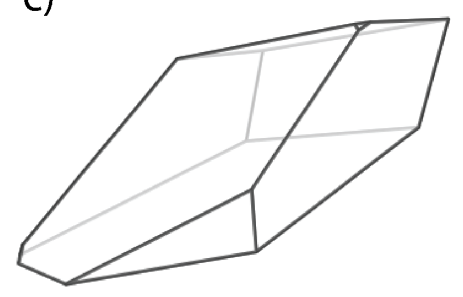

b)

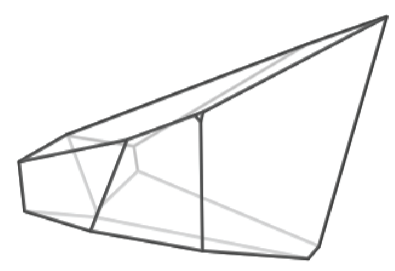

d)

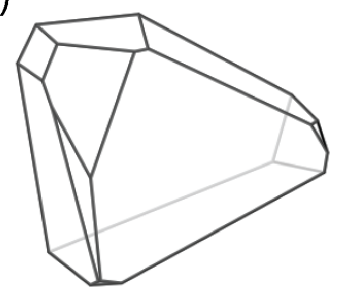

Figure 1: (a) A typical nanocrystalline structure created by the Poisson Voronoi method and examples of (b) grains with sharp points (c) wedge-shaped grains and (d) planar prismatic and taken from this structure.

where $f_{\text {grain }}$ is the free energy density, $W_{\phi}$ is a scaling factor and $\epsilon$ is an isotropic GB energy scaling factor. The free energy density is defined as

$$
f_{\text {grain }}=\frac{4}{3}\left[1-4 \sum_{i=1}^{n_{\phi}} \phi_{i}^{3}+3\left(\sum_{i=1}^{n_{\phi}} \phi_{i}^{2}\right)^{2}\right] .
$$

Minima of $f_{\text {grain }}$ are conveniently located where $\phi_{i}=1, \phi_{j}=0 \forall i \neq j$. The spatial and temporal evotion of OPs is defined by the Allen-Cahn equation [67]:

$$
\frac{\partial \phi_{i}}{\partial t}=-L\left(\frac{\delta \mathcal{F}_{t o t}}{\delta \phi_{i}}\right),
$$

where $L$ is a grain boundary mobility factor. In this work, simulations were performed on a $120 \times 120 \times 120$ voxel cube with 100 order parameters with an initial seed of uniformly distributed random noise. Snapshots were taken within the topologically self-similar regime.

\subsection{Atomistic Structure Generation}

80

Phase field simulation data was converted to atomistic structures, here called Discrete Phase Field (DPF) structures. Individual grains were extracted from each order parameter using a 
cluster labelling algorithm [68]. The atomistic grains within the microstructure were then created by processing each grain order parameter using the following algorithm:

1. Replicate the order parameter in any direction where it crosses a periodic boundary.

2. Identify contiguous regions using a burn algorithm.

3. Choose a contiguous region that contains voxels in the original box and fill this region with atoms by enumerating integer multiples of lattice vectors. Lattice orientations are assigned randomly according to a uniform distribution.

4. Check that each atom is in a position with $\phi>\phi_{\text {cutoff }}$ where $\phi \in[0,1]$ is the interpolated value of the order parameter at the atom position and $\phi_{\text {cutoff }}$ is a tunable parameter. Interpolation is necessary for the production of smooth grain boundaries.

This algorithm allows for lattice orientation to be separately defined for grains that originally shared an order parameter, while also ensuring that assigned lattices are contiguous across periodic boundaries. A three-dimensional microstructure generated from the phase field model and corresponding atomistic microstructure are presented in Figure 2.

The order parameter cutoff, $\phi_{\text {cutoff }}$, allows for the control of the density of the structure. At $\phi_{\text {cutoff }}=0.5$, ellipsoid grains with common tangents are formed, leaving voids at triple junctions and vertex nodes, which are not removed through low temperature equilibration. Reducing $\phi_{\text {cutoff }}$ inflates the grains which results in overlap at grain boundaries and fills triple junctions. Deleting atoms within $90 \%$ of the first nearest neighbor distance produces structures with stable density after $10 \mathrm{ps}$ equilibration at $10 \mathrm{~K}$. $\phi_{\text {cutoff }}=0.35$ was found to be the highest cutoff that produced structure with total density equivalent to those produced by the PV method after short, low temperature, equilibration suggesting that both triple junctions and grain boundaries had relaxed to reasonably dense configurations.

Five realizations of atomistic microstructures were generated using the DPF and PV methods in order to obtain a more statistical sense of the results. Generated microstructures had simulation box sizes of $(50 \mathrm{~nm})^{3} \pm 1 \%$, composed of around 10 million atoms. Microstructures were generated with an average grain diameter of 10nm using the spherical grain approximation.

Due to the diffuse nature of interfaces within the phase field framework, faces were counted by identifying voxels with multiple non-zero order parameters. It was assumed that these order parameters must then be connected by faces. Isosurfaces at $\phi=\phi_{\text {cutoff } f}$ were extracted for each grain, from which the surface area and volume were calculated. Since some atoms are deleted from each grain, this overestimates the volume and surface area of each grain, but remains a useful first order approximation. 

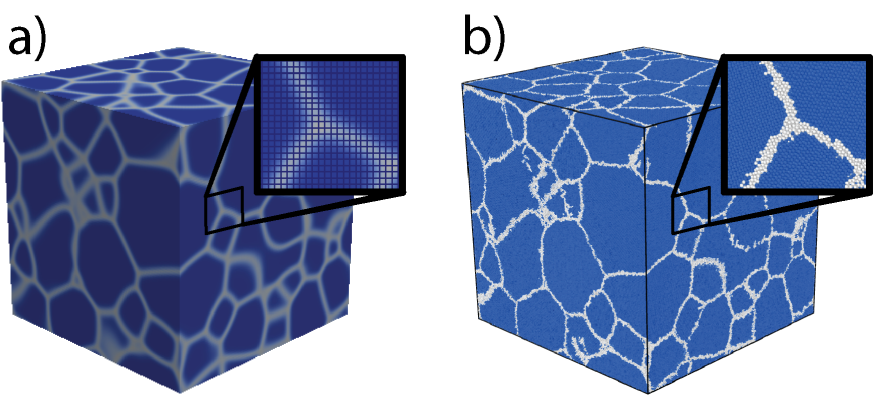

Figure 2: Microstructures generated from (a) Phase Field and (b) Discrete Phase Field. In all images GBs are white while grains are blue. GBs are identified by $\sum \phi_{i}^{2}\left(1-\phi_{i}\right)^{2}$ in (a) and by common neighbor analysis in (b).

The volume and surface area of the five structures created using the PV tessellation were computed using the Voro++ Library [69]. Calculated values were found to be consistent with the surface extraction method performed on blurred digital Voronoi structures.

\subsection{Molecular Dynamics Simulation}

MD simulations were performed using an embedded atom method (EAM) interatomic potential for nickel [70] using the LAMMPS MD package [71]. All simulations employed threedimensional periodic boundary conditions. For each structure a conjugate gradient minimization was performed, allowing the simulation box to relax to a target pressure of zero in each dimension, followed by equilibration for $20 \mathrm{ps}$ at $10 \mathrm{~K}$ and at $300 \mathrm{~K}$ with zero pressure maintained using the isothermal-isobaric (NPT) ensemble. Uniaxial tension up to $10 \%$ was performed along the $\mathrm{x}$-axis of each sample at a strain rate of $10^{9} \mathrm{~s}^{-1}$ at $300 \mathrm{~K}$, while the normal pressure was maintained at zero in the transverse directions.

\subsection{Kinematic Metrics}

To analyze the deformation behavior of the system, the continuum strain field is approximated at each atom using the method described by Tucker et al. [72, 73, 74, 75]. The deformation gradient tensor, $\mathbf{F}$, is approximated at each atom by computing the change of its initial neighborhood. The strain tensor is obtained from the deformation gradient tensor as

$$
\mathbf{E}=\frac{1}{2}\left(\mathbf{F}^{\mathrm{T}} \mathbf{F}-\mathbf{I}\right)
$$

where $\mathbf{I}$ is the identity tensor. The strain tensor is transformed into two scalar metrics: dilatation, $\delta$, and von Mises strain, $\epsilon_{v m}$, defined by:

$$
\delta=\mathrm{E}_{i i}
$$




$$
\epsilon_{v m}=\sqrt{\frac{3}{2} \mathrm{E}_{i j} \mathrm{E}_{i j}}
$$

The microrotation vector, $\varphi_{k}$, is also computed from the deformation gradient as the skew part of the rotation matrix, $\mathbf{R}$ obtained from the polar decomposition of $\mathbf{F}$ :

$$
\begin{gathered}
\mathbf{F}=\mathbf{R U} \\
\varphi_{k}=\epsilon_{i j k} \operatorname{skew}(\mathbf{R})_{i j}
\end{gathered}
$$
matching other thermodynamically driven grain growth methods [79], while the PV method produces a lognormal-like distribution. The surface area distribution is notably different for the 

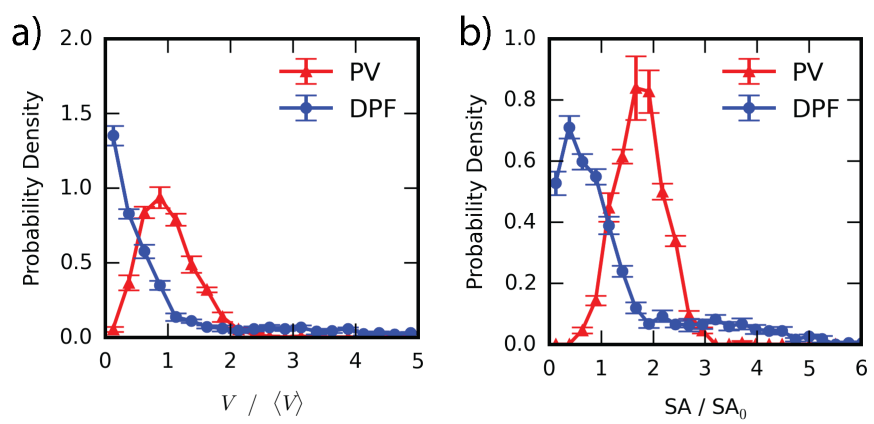

Figure 3: Characterization of grain topology in Poisson Voronoi (PV) and Discrete Phase Fields (DPF) by (a) normalized grain volume distribution (b) surface area distribution, were $\mathrm{SA}_{0}$ is the surface or a $10 \mathrm{~nm}$ diameter sphere. Error bars are the standard error of the mean across the five instances. The dotted line in panel (b) indicates the surface area of a $10 \mathrm{~nm}$ diameter sphere.
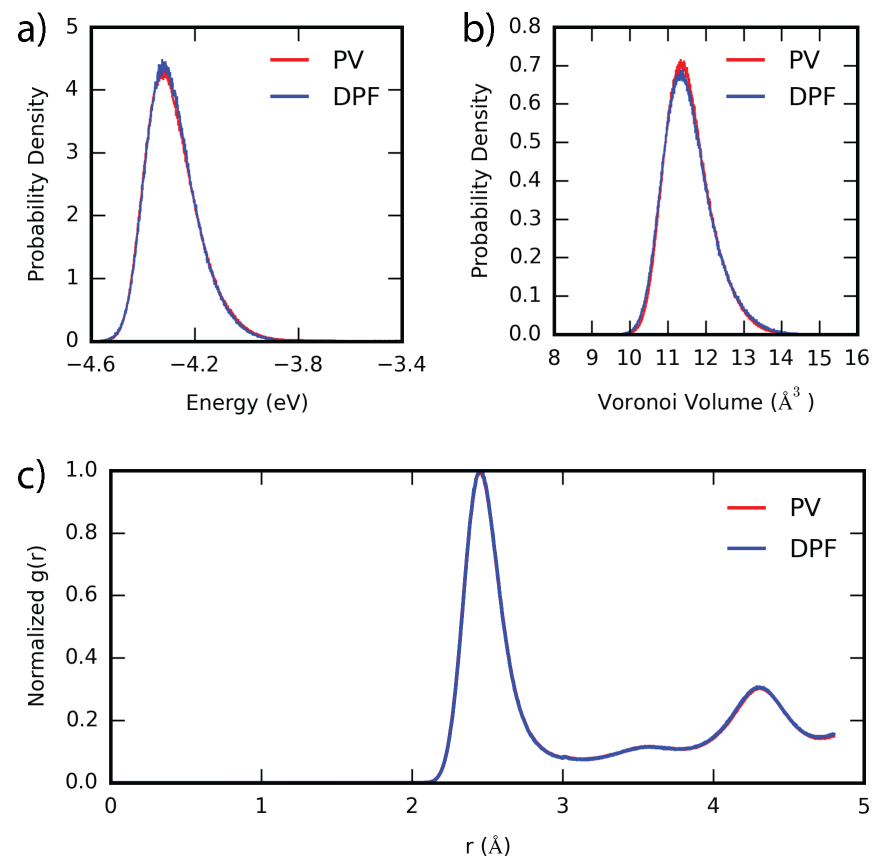

Figure 4: Per-atom properties of defect atoms (a) Atomic energy histogram (b) Voronoi volume histogram (c) Normalized radial distribution function 


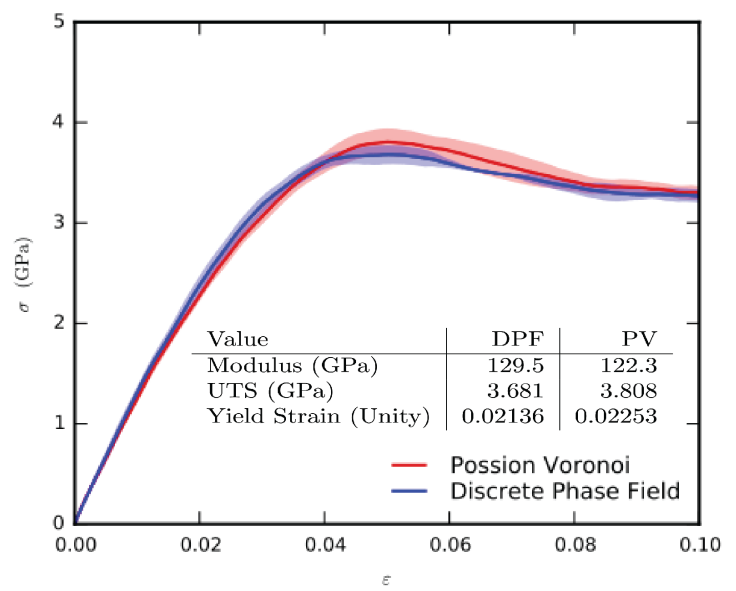

Figure 5: Mean mechanical response under uniaxial tension for structures produced by the three methods. Compared to the Discrete Phase Field, the Poisson Voronoi over estimates tensile strength. Lighter areas indicate a single standard deviation from the mean stress. The inset table shows common values associated with each stress strain curve: elastic modulus, ultimate tensile stress (UTS) and $0.02 \%$ offset yield strain.

DPF structures; the large number of small grains results in a peak below the spherical 10nm grain average, while the PV structure has a peak at a larger surface area. To quantify the difference in grain boundary structure, the distributions of the per-atom potential energy and volume of the resultant cells created by Voronoi tessellation, or Voronoi volume, were computed for defect atoms, in addition to the radial distribution function (Fig. 4). All profiles were quite similar, but some small differences were noted. The energy profile of the DPF structures is slightly more narrow and the PV structures contain significantly more atoms at the high end of the distribution, though still a very small fraction of total atoms. The Voronoi volume profile shows the opposite trend; the PV distribution is more narrow. The radial distribution function for both structures is exactly identical. Based on this analysis, the distribution of local atomic structure does not appear to differ significantly between the two methods.

Despite these differences in structure, the mean mechanical response of structures generating using the two methods is similar (Figure 5). While there are substantial differences in both the grain volume and surface area distributions produced by the two methods, these differences do not have a significant effect on elastic modulus, ultimate tensile strength and $0.2 \%$ offset yield strain (Figure 5 inset table). While macroscopic mechanical responses of polycrystals obtained from two approaches agree relatively well, significantly different local responses are observed. Figure 6 shows development of dislocation and twin groups upon $6 \%$ deformation. Qualitatively, 
a)

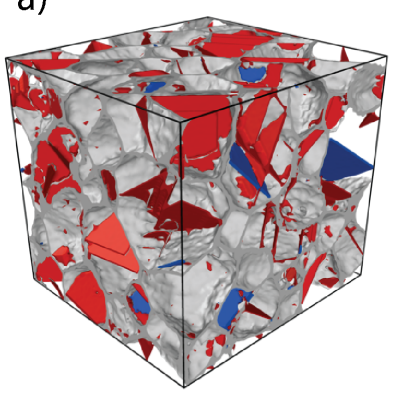

b)

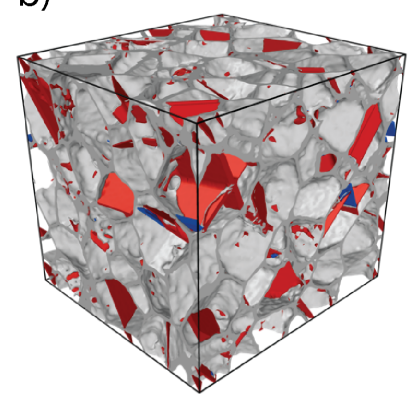

Figure 6: Paths of dislocations (red) and twins (blue) in the grain boundary network (gray) in single instances of (a) DPF and (b) PV Structures at 6\% uniaxial tensile strain. A significantly higher number of both dislocations and twins have been generated in DPF compared to PV, suggesting the fundamental role of GB structure on deformation behavior.
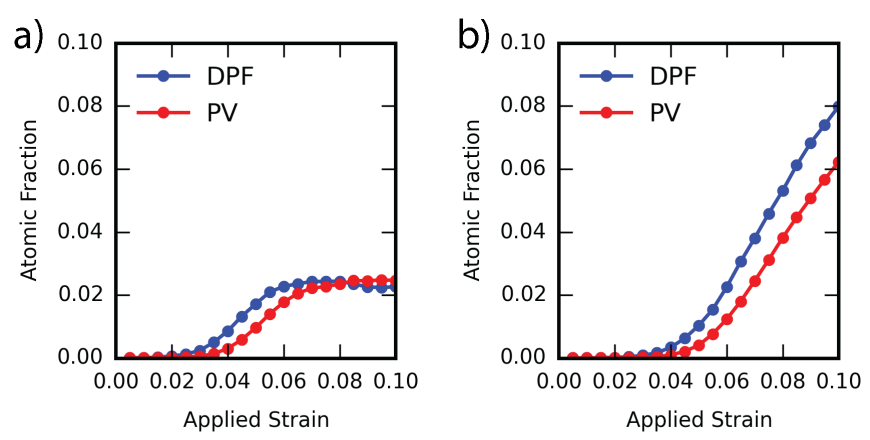

Figure 7: Fraction of atoms that have undergone (a) partial slip and (b) perfect slip as a function of imposed tensile strain for DPF (blue) and PV (red) microstructures.

a much higher number of dislocations appear to be present in the DPF structures. This disparity is confirmed through quantitative analysis of dislocation slip. Figure 7 shows the fraction of atoms that have undergone partial or perfect slip during deformation. In both cases, while dislocation activity increases during deformation, the DPF structure shows a higher fraction of slipped atoms at a given strain. This suggests that in PV structures, the nucleation of dislocations is inhibited relative to the DPF structure, requiring either a higher dislocation nucleation energy or the presence of a lower-cost path, such as GB sliding, in the PV structure.

Differences in the operative GB and dislocation mechanisms at a given strain can be directly linked to the fundamentally different atomic structure of interfaces generated by the two methods. PV methods tend to create perfectly flat GBs, while the DPF methods creates more realistic 
curved GBs. It is likely that the planar nature of interfaces and unrealistic geometric constraints within the microstructures from PV facilitates GB sliding and reordering, while the curved GBs inhibit this strain accommodation mechanism. Furthermore, the increased volume fraction of GBs induced by curvature in the DPF structures may result in an increased number of sites for dislocation nucleation, leading to higher dislocation concentrations at lower strains compared to PV structures. Finally, dislocations occasionally nucleate at triple junctions in the DPF structures, but this behavior is rarely observed in the PV structures, suggesting that the character of triple junctions may also play a role in dislocation plasticity. Further study of these systems is required to quantitatively resolve the contributions of these additional intercrystalline effects.

Figure 8 shows the fraction of strain metrics accommodated by each of four deformation mechanisms: elastic strain in the bulk, grain boundary sliding and restructuring, dislocation slip and twinning. The strain is quantified according to three kinematic metrics (a) $E_{11}$, the strain component parallel to the axis of loading, (b) absolute dilatation, and (c) Von Mises Equivalent strain. The data shown in Figure 8 is obtained by summing the relevant kinematic metric for every atom within each group (or mechanism) and then normalizing by the total strain tensor of all atoms in the simulation. As such, $E_{11}$ for the GB group in Figure ?? is found by summing the $E_{11}$ strain tensor component from each atom in the GB group to obtain a total $E_{11}$ value and then normalizing by the total $E_{11}$ summing over all atoms in the simulation cell, as detailed earlier. In all cases, strain is initially dominated by elastic deformation in the bulk which gradually gives way to GB-mediated deformation at higher strains. Beyond approximately $4 \%$ strain, dislocations and twins become the dominant deformation modes, further decreasing the amount of strain accommodated in the bulk. 
a)

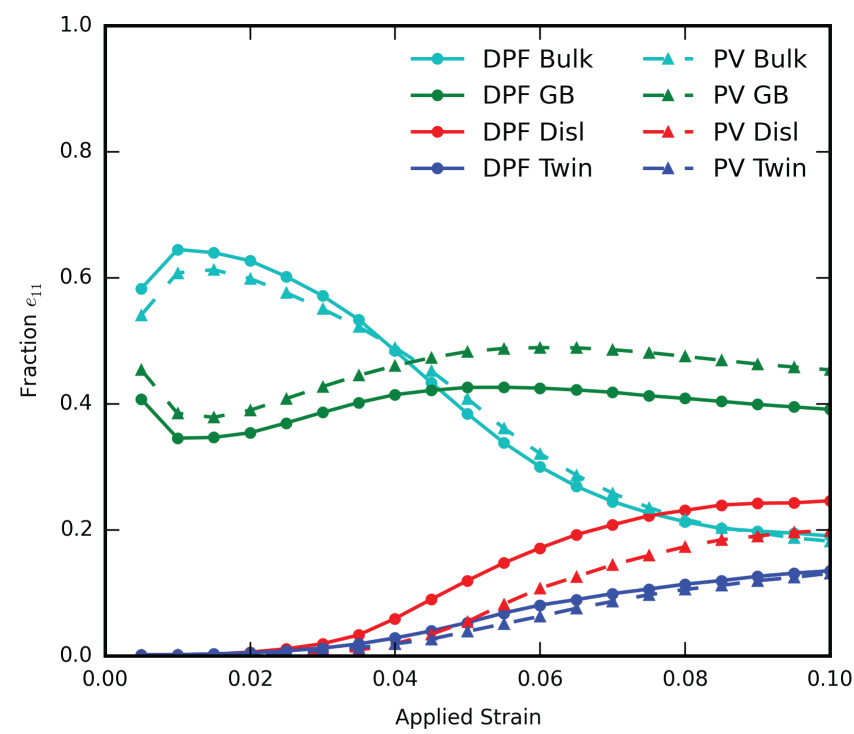

c)

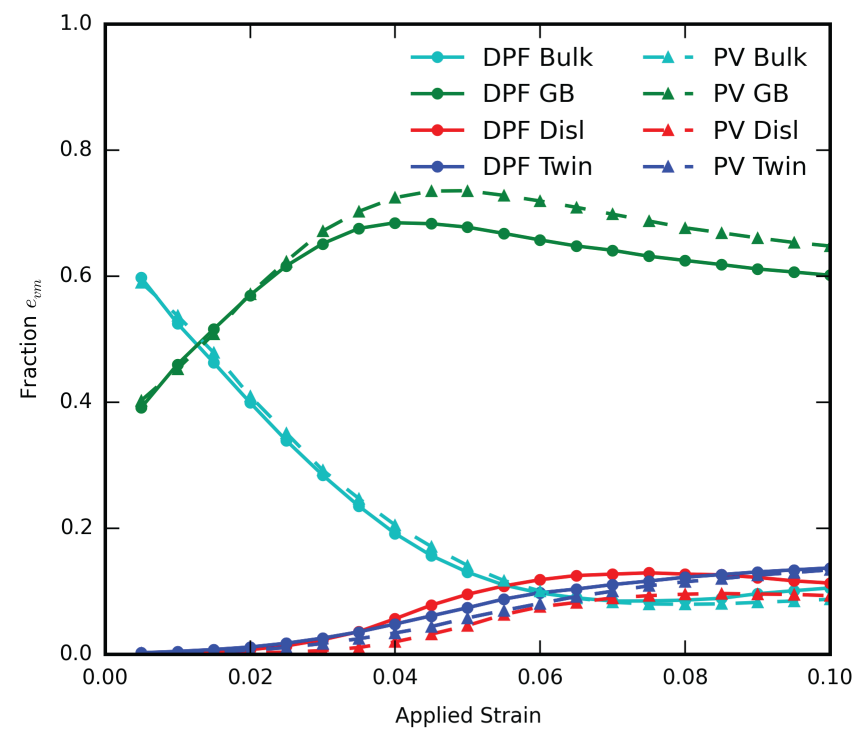

e)

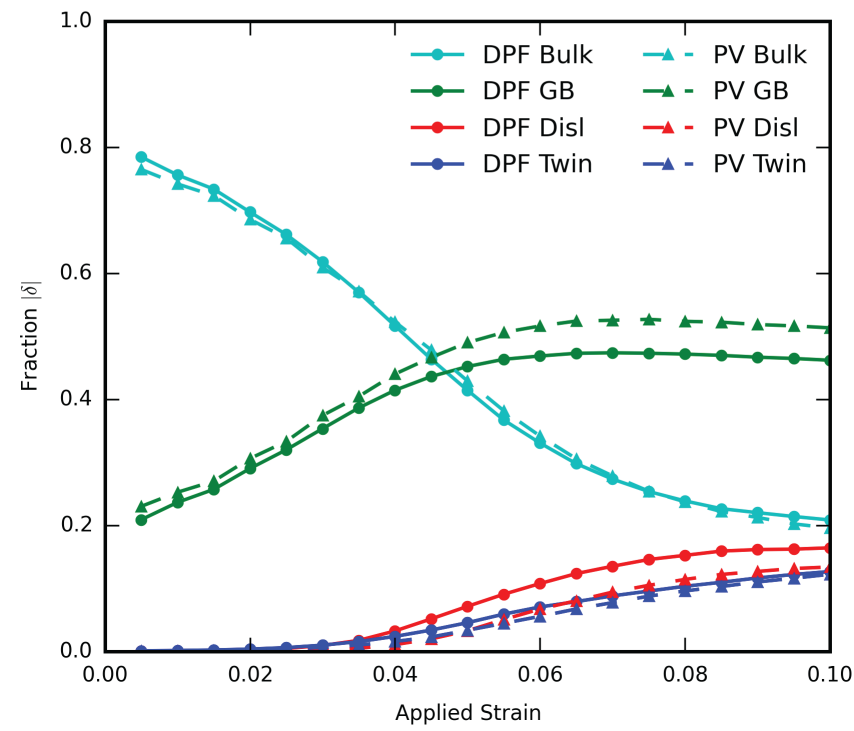

a)

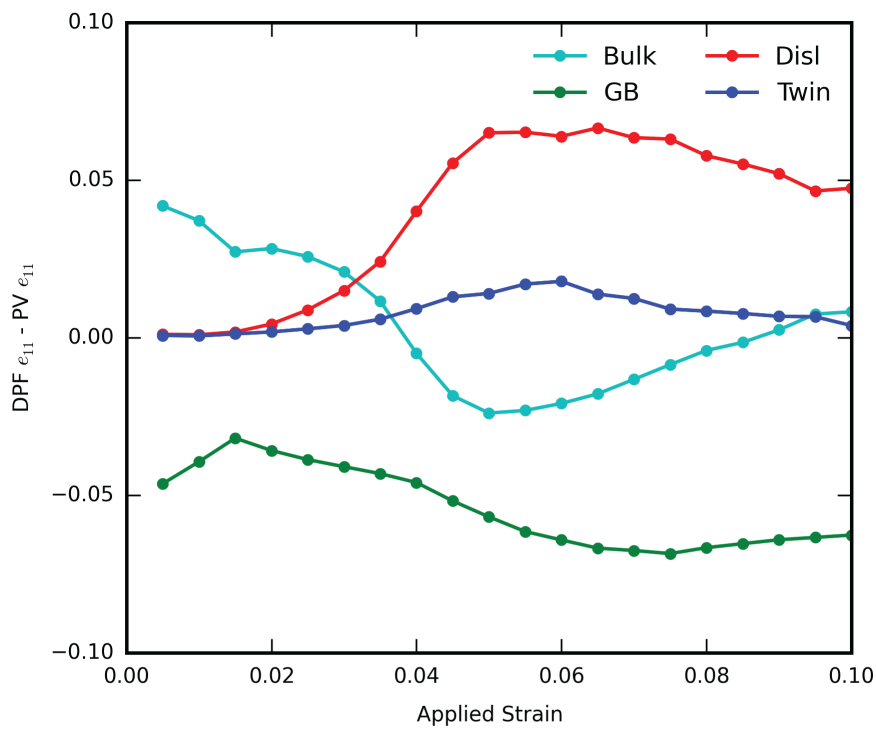

c)

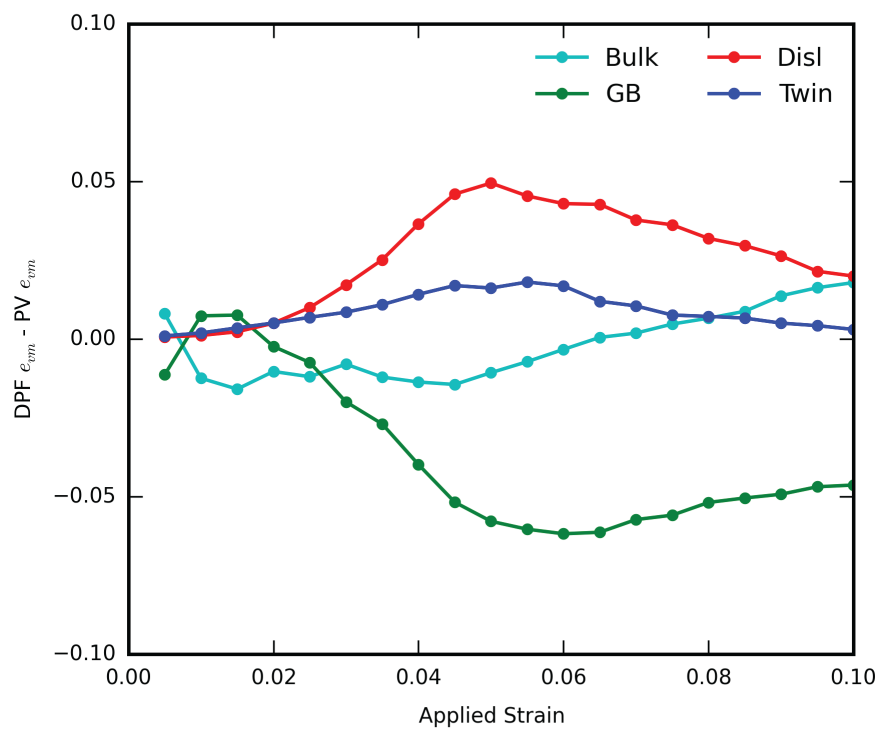

e)

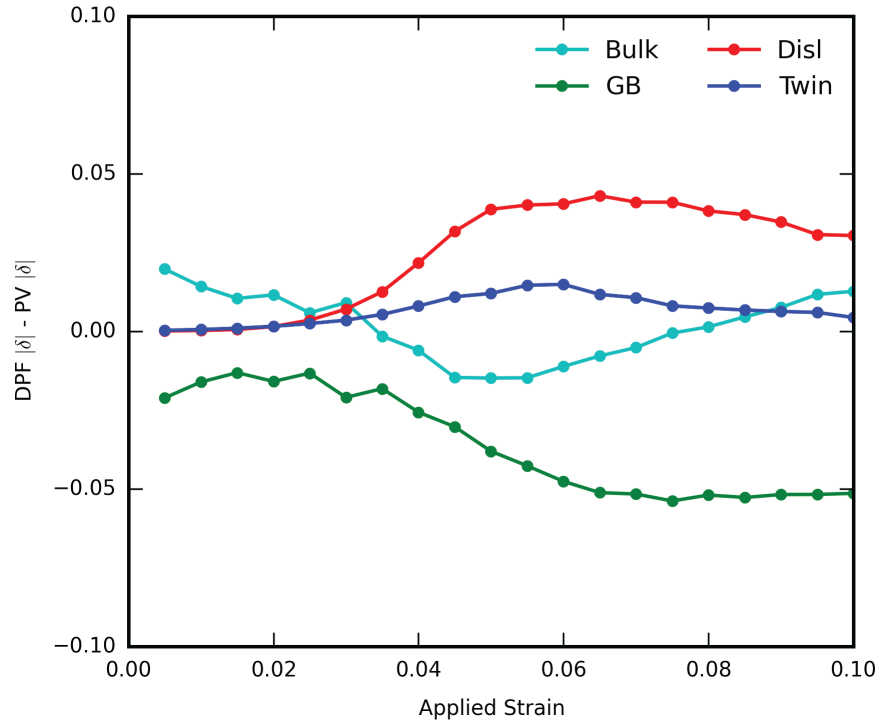

Figure 8: (left) The fraction of total strain accommodated by bulk elastic deformation, GB-mediated deformation, dislocation plasticity and twinning, and (right) the difference in fraction between the DPF and PV structures for each mechanism, with strain quantified using (a,b) $E_{11}$ $(\mathrm{c}, \mathrm{d})$ Absolute Dilatation and (e,f) Von Mises Equivalent strain. 
The differences between DPF and PV structures show that, for all three metrics, a higher fraction of strain is accommodated by GBs in PV structures, while DPF structures exhibit a larger contribution from dislocation plasticity. The differences in GB structure outlined previously and their corresponding effect on dislocation nucleation and GB sliding produce significant differences in the competition of operative deformation mechanisms. In all three metrics, the DPF structures show a significantly higher fraction of strain accommodated by dislocations offset primarily by a decreased contribution from GBs, supporting the idea that GB deformations are inhibited in the DPF structures. While the fraction of strain accommodated by twinning is stable, that accommodated by bulk atoms is not. As more atoms slip due to passing dislocations, they move from the bulk group to the dislocation group, giving rise to a disparity in calculated elastic strain accommodation between the two sets of structures. Future work should resolve the elastic and plastic components of individual atomic strain, rather than assuming all deformation is either one or the other.

The distribution of strain mechanisms raises some other interesting questions. Figure ?? exhibits a small transient effect in the first time step that is not present in other kinematic metrics. This is likely the result of strain-induced restructured of unstable GBs. It is unclear, however, why only $E_{11}$ captures this effect. All metrics also show a peak in the difference of strain accommodation of twins near $6 \%$ strain, which suggests that GB character also plays a role in twin nucleation. Also present are significant changes in the difference in strain accommodation by bulk atoms, which in all kinematic metrics shows significant trends towards the DPF structure beyond $6 \%$ strain. This may be the result of differences in GB migration, which is not individually resolved in this study.

\section{Conclusion}

Molecular dynamics simulations of nanocrystalline solids typically employ Poisson Voronoi methods to create interfaces and grains, commonly used to study the mechanical properties of nanocrystalline systems. In this work, we present a novel method for the creation of nanocrystalline structures from phase field simulations. This method has numerous advantages over Voronoi methods in that it produces curved, rather than flat boundaries, recovers the correct triple junction angle with greater accuracy and produces grain size and surface area distributions more consistent with other grain growth models. The mean mechanical response of structures generated using both methods is similar despite differences in grain size and surface area distributions, however, probing the deformation mechanisms of both structures reveals significant 
differences. Structures generated using the phase field based method displayed a greater tendency towards dislocation slip rather than grain boundary sliding. Extensive study has been performed on the transition from Hall-Petch behavior in coarse-grained structures to grain boundary mediated deformation in nanocrystalline structures, however, that work has relied on the Voronoi tessellation method to produce microstructures. The work presented here demonstrates that the dislocation plasticity in these systems is sensitive to interface character. Therefore, grain size quantifications of the Hall-Petch breakdown performed on microstructures generated using Voronoi tessellation method are likely inaccurate and must be reevaluated with greater consideration of interface character.

\section{Acknowledgements}

Sandia National Laboratories is a multi-program laboratory managed and operated by Sandia Corporation, a wholly owned subsidiary of Lockheed Martin Corporation, for the US Department of Energys National Nuclear Security Administration under contract DE-AC04-94AL85000. This material is based on work partially supported by the National Science Foundation under Grant No. DMR-1410970.

\section{References}

[1] Jason R. Trelewicz and Christopher A. Schuh. Grain boundary segregation and thermodynamically stable binary nanocrystalline alloys. Physical Review B - Condensed Matter and Materials Physics, 79(9):94112, 2009.

[2] H Gleiter. Nanocrystalline materials. Progress in Materials Science, 33(4):223-315, 1989.

[3] R. Birringer. Nanocrystalline materials. Materials Science and Engineering: A, 117:33-43, sep 1989.

[4] Lei Lu, Yongfeng Shen, Xianhua Chen, Lihua Qian, and K. Lu. Ultrahigh Strength and High Electrical Conductivity in Copper. Science, 304(5669):422-426, apr 2004.

[5] M. A. Meyers, A. Mishra, and D. J. Benson. Mechanical properties of nanocrystalline materials. Progress in Materials Science, 51(4):427-556, may 2006.

[6] A. H. Chokshi, A. Rosen, J. Karch, and H. Gleiter. On the validity of the hall-petch relationship in nanocrystalline materials. Scripta Metallurgica, 23(10):1679-1683, oct 1989. 
[7] C. E. Carlton and P. J. Ferreira. What is behind the inverse HallPetch effect in nanocrystalline materials? Acta Materialia, 55(11):3749-3756, jun 2007.

[8] F Ebrahimi, G.R Bourne, M.S Kelly, and T.E Matthews. Mechanical properties of nanocrystalline nickel produced by electrodeposition. Nanostructured Materials, 11(3):343-350, may 1999.

[9] O ERTORER, T TOPPING, Y LI, W MOSS, and E LAVERNIA. Enhanced tensile strength and high ductility in cryomilled commercially pure titanium. Scripta Materialia, 60(7):586589, apr 2009.

[10] G.D. Hughes, S.D. Smith, C.S. Pande, H.R. Johnson, and R.W. Armstrong. Hall-petch strengthening for the microhardness of twelve nanometer grain diameter electrodeposited nickel. Scripta Metallurgica, 20(1):93-97, jan 1986.

[11] D. Jang and M. Atzmon. Grain-size dependence of plastic deformation in nanocrystalline Fe. Journal of Applied Physics, 93(11):9282, may 2003.

[12] A Karimpoor. High strength nanocrystalline cobalt with high tensile ductility. Scripta Materialia, 49(7):651-656, oct 2003.

[13] E.W. Qin, L. Lu, N.R. Tao, J. Tan, and K. Lu. Enhanced fracture toughness and strength in bulk nanocrystalline $\mathrm{Cu}$ with nanoscale twin bundles. Acta Materialia, 57(20):6215-6225, dec 2009 .

[14] Haofei Zhou and Shaoxing Qu. The effect of nanoscale twin boundaries on fracture toughness in nanocrystalline Ni. Nanotechnology, 21(3):035706, jan 2010.

[15] H. A. Padilla and B. L. Boyce. A Review of Fatigue Behavior in Nanocrystalline Metals. Experimental Mechanics, 50(1):5-23, oct 2009.

[16] Dong-Cheon Baek and Soon-Bok Lee. Fatigue Behavior of Electrodeposited Nanocrystalline Nickel Films. Procedia Engineering, 10:3006-3011, 2011.

[17] N.A. Mara, A.V. Sergueeva, T.D. Mara, S.X. McFadden, and A.K. Mukherjee. Superplasticity and cooperative grain boundary sliding in nanocrystalline Ni3Al. Materials Science and Engineering: A, 463(1-2):238-244, aug 2007.

[18] M.J.N.V. Prasad and A.H. Chokshi. Superplasticity in electrodeposited nanocrystalline nickel. Acta Materialia, 58(17):5724-5736, oct 2010. 
[19] S. X. McFadden, R. S. Mishra, R. Z. Valiev, A. P. Zhilyaev, and A. K. Mukherjee. Lowtemperature superplasticity in nanostructured nickel and metal alloys. 398(6729):684-686, apr 1999.

[20] Ruslan Valiev. Nanostructuring of metals by severe plastic deformation for advanced properties. Nature materials, 3(8):511-6, aug 2004.

[21] M. Samaras, W. Hoffelner, and M. Victoria. Irradiation of pre-existing voids in nanocrystalline iron. Journal of Nuclear Materials, 352(13):50-56, jun 2006.

[22] Graeme Ackland. Controlling Radiation Damage. Science, 327(5973):1587-1588, 2010.

[23] A. Misra, M. J. Demkowicz, X. Zhang, and R. G. Hoagland. The radiation damage tolerance of ultra-high strength nanolayered composites. JOM, 59(9):62-65, sep 2007.

[24] J. H Driver. Stability of nanostructured metals and alloys. Scripta Materialia, 51(8):819823 , oct 2004 .

[25] Riccardo Ferrando, Julius Jellinek, and Roy L. Johnston. Nanoalloys: From theory to applications of alloy clusters and nanoparticles. Chemical Reviews, 108(3):845-910, mar 2008.

[26] R. A. Andrievski. Review of thermal stability of nanomaterials. Journal of Materials Science, 49(4):1449-1460, 2013.

[27] E O Hall. The Deformation and Ageing of Mild Steel: III Discussion of Results. Proceedings of the Physical Society. Section B, 64(9):747-753, sep 1951.

[28] N.J. Petch. The Cleavage Strength of Polycrystals. J. Iron Steel Inst, 174:25 - 28, 1953.

[29] Jakob Schiøtz, Francesco D Di Tolla, Karsten W Jacobsen, J Schiotz, Francesco D Di Tolla, and Karsten W Jacobsen. Softening of nanocrystalline metals at very small grain sizes. Nature, 391(6667):561-563, 1998.

[30] Hans Conrad. Grain-size dependence of the flow stress of $\mathrm{Cu}$ from millimeters to nanometers. Metallurgical and Materials Transactions A, 35(9):2681-2695, sep 2004.

[31] Vesselin Yamakov, Dieter Wolf, Simon R Phillpot, Amiya K Mukherjee, and Herbert Gleiter. Dislocation processes in the deformation of nanocrystalline aluminium by moleculardynamics simulation. Nature Materials, 1(1):45-49, 2002. 
[32] V Yamakov, D Wolf, S R Phillpot, A K Mukherjee, and H Gleiter. Deformation-mechanism map for nanocrystalline metals by molecular-dynamics simulation. Nature Materials, $3(1): 43-47,2004$.

[33] Jakob Schiøtz and Karsten W Jacobsen. A maximum in the strength of nanocrystalline copper. Science (New York, N.Y.), 301(5638):1357-9, sep 2003.

[34] M.A. Tschopp and D.L. McDowell. Grain boundary dislocation sources in nanocrystalline copper. Scripta Materialia, 58(4):299-302, feb 2008.

[35] H Van Swygenhoven and A Caro. Plastic behavior of nanophase Ni: A molecular dynamics computer simulation. Applied Physics Letters, 71(12):1652-1654, 1997.

[36] Helena Van Swygenhoven and Julia R. Weertman. Deformation in nanocrystalline metals. Materials Today, 9(5):24-31, may 2006.

[37] Rahul K. Rajgarhia, Douglas E. Spearot, and Ashok Saxena. Molecular Dynamics Simulations of Dislocation Activity in Single-Crystal and Nanocrystalline Copper Doped with Antimony. Metallurgical and Materials Transactions A, 41(4):854-860, feb 2010.

[38] Timothy J. Rupert and Christopher A. Schuh. Mechanically driven grain boundary relaxation: a mechanism for cyclic hardening in nanocrystalline Ni. Philosophical Magazine Letters, 92(1):20-28, jan 2012.

[39] H. N. Jarmakani, E. M. Bringa, P. Erhart, B. A. Remington, Y. M. Wang, N. Q. Vo, and M. A. Meyers. Molecular dynamics simulations of shock compression of nickel: From monocrystals to nanocrystals. Acta Materialia, 56(19):5584-5604, nov 2008.

[40] Meizhen Xiang, Haibo Hu, and Jun Chen. Spalling and melting in nanocrystalline $\mathrm{Pb}$ under shock loading: Molecular dynamics studies. Journal of Applied Physics, 113(14):144312, apr 2013.

[41] Sheng-Nian Nian Luo, Timothy C. Germann, Tapan G. Desai, Davis L. Tonks, and Qi An. Anisotropic shock response of columnar nanocrystalline $\mathrm{Cu}$. Journal of Applied Physics, 107(12):123507, 2010.

[42] Xian-Ming M Bai, Arthur F Voter, Richard G Hoagland, Michael Nastasi, and Blas P Uberuaga. Efficient Annealing of Radiation Damage Near Grain Boundaries via Interstitial Emission. Science, 327(5973):1631-1634, 2010. 
[47] Huicong Dong, Bin Wen, and Roderick Melnik. Relative importance of grain boundaries and size effects in thermal conductivity of nanocrystalline materials. Scientific reports, 4:7037, nov 2014.

[48] D. F. Watson. Computing the n-dimensional Delaunay tessellation with application to

53] W. W. Mullins. TwoDimensional Motion of Idealized Grain Boundaries. Journal of Applied Physics, 27(8):900-904, 1956.

[54] W. Z. Han, M. J. Demkowicz, E. G. Fu, Y. Q. Wang, and A. Misra. Effect of grain boundary character on sink efficiency. Acta Materialia, 60(18):6341-6351, oct 2012. 
[55] H. Van Swygenhoven and P. M. Derlet. Grain-boundary sliding in nanocrystalline fcc metals. Physical Review B, 64(22):224105, nov 2001.

[56] H. Van Swygenhoven, P.m. M. Derlet, and A. Hasnaoui. Atomistic modeling of strength of nanocrystalline metals. Advanced Engineering Materials, 5(5):345-350, may 2003.

[57] H. Van Swygenhoven, P.M. Derlet, and A.G. Frøseth. Nucleation and propagation of dislocations in nanocrystalline fcc metals. Acta Materialia, 54(7):1975-1983, apr 2006.

[58] T. Xu and M. Li. Topological and statistical properties of a constrained Voronoi tessellation. Philosophical Magazine, 89(4):349-374, feb 2009.

[59] Yanhong Shen, Tao Gao, Xiaofeng Tian, Xiaojun Chen, ChengJian Xiao, and Tiecheng Lu. Constructing three-dimensional (3D) nanocrystalline models of Li4SiO4 for numerical modeling and simulation. Scientific Reports, 5, jun 2015.

[60] F. Fritzen, T. Böhlke, and E. Schnack. Periodic three-dimensional mesh generation for crystalline aggregates based on Voronoi tessellations. Computational Mechanics, 43(5):701$713,2008$.

[61] Z. Y. Hou, Z. A. Tian, Y. F. Mo, R. S. Liu, J. G. Wang, X. M. Shuai, and K. J. Dong. Atomic dynamics of grain boundaries in bulk nanocrystalline aluminium: A molecular dynamics simulation study. Computational Materials Science, 108, Part:177-182, oct 2015.

[62] K. Kadau, P. S. Lomdahl, B. L. Holian, T. C. Germann, D. Kadau, P. Entel, D. E. Wolf, M. Kreth, and F. Westerhoff. Molecular-dynamics study of mechanical deformation in nano-crystalline aluminum. Metallurgical and Materials Transactions A, 35(9):2719-2723, sep 2004 .

[63] C. E. Krill III and L. Q. Q Chen. Computer simulation of 3-D grain growth using a phasefield model. Acta Materialia, 50(12):3059-3075, jul 2002.

[64] D. Fan and L.-Q. Chen. Computer simulation of grain growth using a continuum field model. Acta Materialia, 45(2):611-622, feb 1997.

[65] Fadi Abdeljawad and Stephen M. Foiles. Stabilization of nanocrystalline alloys via grain boundary segregation: A diffuse interface model. Acta Materialia, 101:159-171, 2015. 
[66] Fadi Abdeljawad, Benjamin Völker, Ryan Davis, Robert M McMeeking, and Mikko Haataja. Connecting microstructural coarsening processes to electrochemical performance in solid oxide fuel cells: $\{\mathrm{An}\}$ integrated modeling approach. Journal of Power Sources, 250:319331 , mar 2014 .

[67] Samuel M. Allen and John W. Cahn. A microscopic theory for antiphase boundary motion and its application to antiphase domain coarsening. Acta Metallurgica, 27(6):1085-1095, 1979.

[68] Fabian Pedregosa, Gaël Varoquaux, Alexandre Gramfort, Vincent Michel, Bertrand Thirion, Olivier Grisel, Mathieu Blondel, Peter Prettenhofer, Ron Weiss, Vincent Dubourg, Jake Vanderplas, Alexandre Passos, David Cournapeau, Matthieu Brucher, Matthieu Perrot, and Édouard Duchesnay. Scikit-learn: Machine Learning in Python. Journal of Machine Learning Research, 12(Oct):2825-2830, 2011.

[69] Chris H. Rycroft. VORO++: A three-dimensional Voronoi cell library in C++. Chaos, 19(4):41111, 2009.

[70] S Foiles and J Hoyt. Computation of grain boundary stiffness and mobility from boundary fluctuations. Acta Materialia, 54(12):3351-3357, jul 2006.

[71] Steve Plimpton. Fast Parallel Algorithms for Short-Range Molecular Dynamics. Journal of Computational Physics, 117(1):1-19, mar 1995.

[72] G. J. Tucker, J.a. A. Zimmerman, and D. L. McDowell. Shear deformation kinematics of bicrystalline grain boundaries in atomistic simulations. Modelling and Simulation in Materials Science and Engineering, 18(1):15002, jan 2010.

[73] Garritt J. Tucker, Jonathan A. Zimmerman, and David L. McDowell. Continuum metrics for deformation and microrotation from atomistic simulations: Application to grain boundaries. International Journal of Engineering Science, 49(12):1424-1434, dec 2011.

[74] Garritt J Tucker, Shreevant Tiwari, Jonathan A Zimmerman, and David L McDowell. Investigating the deformation of nanocrystalline copper with microscale kinematic metrics and molecular dynamics. Journal of the Mechanics and Physics of Solids, 60(3):471-486, mar 2012 . 
[75] Garritt J. Tucker and Stephen M. Foiles. Quantifying the influence of twin boundaries on the deformation of nanocrystalline copper using atomistic simulations. International Journal of Plasticity, 65:191-205, feb 2015.

[76] Daniel Faken and Hannes Jónsson. Systematic analysis of local atomic structure combined with 3D computer graphics. Computational Materials Science, 2(2):279-286, 1994.

[77] Helio Tsuzuki, Paulo S Branicio, and José P Rino. Structural characterization of deformed crystals by analysis of common atomic neighborhood. Computer Physics Communications, 177(6):518-523, sep 2007.

[78] J. A. Zimmerman, C. L. Kelchner, P. A. Klein, J. C. Hamilton, and S. M. Foiles. Surface Step Effects on Nanoindentation. Physical Review Letters, 87(16):165507, oct 2001.

[79] Emanuel A Lazar, Jeremy K Mason, Robert D MacPherson, and David J Srolovitz. A more accurate three-dimensional grain growth algorithm. Acta Materialia, 59(17):6837-6847, oct 2011. 